\title{
Problems and Complications During the Treatment of Infertility in Women with Polycystic Ovary Syndrome
}

\author{
Müberra NAMLI KALEM1, Ziya KALEM², Timur GÜRGAN² \\ Ankara, Turkey
}

\begin{abstract}
Polycystic ovary syndrome (PCOS) is the most frequent endocrine disorder existing in women in their reproductive years and it is one of the most evaluated and discussed subjects of reproductive medicine with regard to its diagnosis and treatment.

Patients with PCOS constitute the most difficult population in the management of infertility. The factors that increase the success rates in the treatment of PCOS infertility are: pretreatment changes in life style, dietetic and psychological support, a detailed evaluation of the couple and the appropriate selection of the treatment protocol, a wide-spectrum approach to maintaining ovarian and endometrial synchronization in the management of the cycle, and well-developed laboratory conditions to support embryonic quality. However, even if these conditions are provided, OHSS, cancellations of the cycle, poor oocyte, and embryo qualities, unsuccessful fertilization and implantation, chromosomal abnormalities and early losses may still be experienced.
\end{abstract}

Keywords: Polycystic ovary syndrome, Infertility, Treatment, Complication

Gynecol Obstet Reprod Med 2016;22(2):113-124 DOI: 10.21613/GORM.2016.54

\section{Introduction}

PCOS is a reproductive disorder that occurs in adolescence with anovulation and hyperandrogenemia, and related signs of menstrual disorders, hirsutism, acne, and obesity. These are associated with psychological problems, and infertility and, in the advanced years, the risk of diabetes, cardiovascular diseases and cancers of the reproductive system, also increase (1). Furthermore, it is also accepted as a metabolic dysfunction that can affect women throughout their lives (2). PCOS is the most frequent endocrine disorder existing in women in their reproductive years (1), and it is one of the most evaluated and discussed subjects of reproductive medicine with regard to its diagnosis (3) and treatment (4).

According to the 2003 Rotterdam criteria, the diagnostic properties of PCOS include oligo- anovulation, clinical and biochemical hyperandrogenism and the polycystic appearance of the ovaries in ultrasonography (3). Increased pulsatile GnRH activity, resulting in high LH levels, hyperactivity in the ovarian theca-stromal cells, and hypofunction in the interaction of FSH- granulosa cells, lead to endocrine abnormali-

\footnotetext{
1 Turgut Özal University Faculty of Medicine Department of Obstetrics and Gynecology Ankara. ${ }^{2}$ Gürgan Clinic, Ankara

Address of Correspondence: Müberra Namll Kalem

Turgut Özal University Faculty of

Medicine Department of Obstetrics and

Gynecology Ankara, Turkey

muberranamli@hotmail.com

Submitted for Publication:

22. 02.2016

Accepted for Publication: 23.05. 2016
}

ties in PCOS, which result in ovarian androgen secretion, pauses in follicular development, and the interruption or slowing of the ovarian cyclic rhythm (4).

In the management of infertility in PCOS, assisted reproductive techniques (ART) is a preferable treatment option (5). Problems experienced in ART management, and the therapeutic complications will be presented in this review.

Ovarian hyperstimulation syndrome is the main problem in PCOS patients undergoing IVF. Incidences of OHSS are between $0.5 \%$ and $4 \%$ in the general IVF population, but it may exceed $10 \%$ in PCOS IVF cases (6). OHSS is an iatrogenic complication triggered by hCG. Early OHSS results from the hyperresponsiveness of the ovarium to the hCG applied, or the persistence of blood hCG levels; late OHSS is caused by the endogenous hCG produced by the implanted embryo (7). The following are responsible for the hCG and OHSS relation: supraphysiological production of angiogenic VEGF released from the follicles by the effect of hCG, and changes in the expressions of the VEGF receptors and modulators in the vessel wall, leading to vascular hyperpermeability (8). The possible mechanisms causing increased OHSS rates in the PCOS group are the follicle and oocyte development increasing three-fold in the same protocol compared with the normoovulatory patients (9), increased expression of VEGF mRNA in the hyperthecal stroma (10), increased ovarian size (11), decreased resistance to blood flow in the stimulated ovaries (12), the LH:FSH ratio greater than two, and the LH dominance, damaging androgen conversion to estrogen and leading to an increased tendency to OHSS (13). 
The etiology and pathophysiology of OHSS has not yet been clarified, and thus empirical and symptomatic considerations play a role in the treatment. The foremost goal in the management of OHSS is prediction and prevention. Strategies developed for this aim can be evaluated in three main groups:

1) Evaluation of risk factors that may predict OHSS

- Risk factors related with the patient:

High level of basal AMH (cut-off level $3.36 \mathrm{ng} / \mathrm{mL}$ ) (13)

Increased number of antral follicles (more than 14) (14)

Lower age $(<33$ years) $(13)$

Previous OHSS history (15)

- Risk factors related with the ovarian response:

Number of follicles on the day of hCG administration (more than 18 follicles greater than $11 \mathrm{~mm}$ ) (14) and/or

High E2 level (higher than $5000 \mathrm{ng} / \mathrm{mL}$ ) (16)

Increased VEGF level (17)

Increased inhibin B level (18)

2) Selection of the suitable ovulation induction protocol prior to stimulation

- Lowering of the gonadotropin dose and duration of administration

-Not to administer FSH on the day of hCG (19)

-Mild stimulation protocols (20)

- Using GnRH antagonist protocol $(21,22)$

- Not to use hCG for the support of luteal phase (23)

- In vitro maturation (IVM) (24)

3) Application of methods to prevent OHSS during stimulation

- Cancellation of the cycle (25)

- Freezing of all embryos to be transferred in another cycle (26)

- Coasting (27)

- Using low-dose hCG, GnRH agonist or recombinant LH for trigger $(28,29,30)$

- Administration of antagonist, dopamine agonist or steroid (31)

- Using albumin or IV volume expanders (31)

- Infusion of IV Ca-gluconate $(32,33)$

It has due to the wide individual variations in responses to gonadotropins, and different clinical experiences related to the day of hCG administration, it is very difficult to optimize the treatment protocol and its duration in the PCOS IVF cycles. Various protocols have been experienced so far: a combination of clomiphene and $\mathrm{hMG}, \mathrm{hMG}$, recombinant (r) FSH, GnRH antagonist with hMG or rFSH have been the most widely used protocols. The standard protocol has been accepted as FSH associated with long-term desensitization (4). However, the long protocol has been currently replaced by the antagonist protocol. been an agreed rule not to administer FSH on the day of hCG; however, there is no available study that directly investigates whether this application triggers the OHSS, or not (7). Additional FSH was reported not to exert a positive effect when follicular maturation is achieved sufficiently enough for triggering ovulation, and it may even affect the endometrial receptivity in a negative way (19).

In the mild stimulation protocol, FSH administration is delayed until the mid or late follicular phase. In this protocol, patterns of the early natural cycle may be reflected onto the treatment cycle, leading to the premature LH surge and cancellation of the cycle; GnRH antagonists may save the cycle in this phase. Suppression with GnRH antagonists in mild stimulation protocols may be helpful in the cycles with a high OHSS risk (20).

Suppression with GnRH agonists in the treatment by gonadotropins increases the possibility of OHSS, since the pretreatment suppression of endogenous gonadotropins requires higher doses of exogenous FSH for appropriate stimulation, and the natural cohort is disordered due to pituitary down-regulation; this results in small antral follicles being included in the cycle, instead of their atresia (7). In the antagonist cycles, OHSS risk decreases via different mechanisms of the effect on the pituitary and ovarian receptors, and thus rates of coasting and cycle cancellation are lower compared with the agonistsuppressed cycles $(21,22)$.

In the luteal phase of the controlled ovarian hyperstimulation protocols, high estrogen and progesterone levels cause negative feedback on the pituitary, leading to decreased endogenous LH levels and decreases in implantation success, and thus luteal support is required (23). Administration of progesterone instead of hCG for luteal support in the PCOS groups with high risks of OHSS, are reported to decrease OHSS rates without causing a change in the pregnancy rate or the risk of early losses (24). Although the number of studies available is not yet sufficient, intranasal use of GnRH analogues in repetitive doses for luteal support is now being considered (25).

In vitro maturation (IVM) is a more comfortable method, compared with conventional IVF cycles, requiring less exogenous gonadotropins and minimal monitoring, and it possesses fewer side effects and a lower OHSS risk (26). In this method, oocytes are recruited from the small, unstimulated follicles in the germinal vesicle phase, and they are matured in vitro in culture media. This method was first developed to prevent OHSS in PCOSs, and later studies have reported that this method is both more reliable and simple but occurs with lower pregnancy rates compared with conventional IVF (27). Following the reported success of new GnRH antagonists and GnRH- agonist trigger applications in decreasing the OHSS rates, IVM is no longer recommended for this indication (28).

Cancellation of the cycle is the most certain method in preventing early OHSS (29). In cases of late OHSS, contraception must be strongly recommended to prevent a possible conception even if the cycle has been cancelled in a patient due to a high risk of OHSS. 
Freezing the embryos and storing them to be transferred in an unstimulated or less stimulated cycle, may protect the patient from a late OHSS, which is a more severe form; however, the risk of early OHSS still exists because of hCG administration. In the patients without early OHSS symptoms, it is wise to wait until the fifth day before making a decision, instead of freezing the embryos early, and to make a single blastocyst transfer if the OHSS symtomps still do not exist, or to freeze them all if symtomps do exist, which has been recommended by Papanikolau et al. (14). It is known that implantation rates of frozen embryos are lower than those of the fresh embryos (30).

Coasting is delaying the gonadotropin administration and hCG-stimulation till the high estradiol levels plateau or decrease in cases of excess follicular response to the ovarian stimulation, and it is generally the first approach when hyperstimulation is detected (31). It is reported that it does not decrease the OHSS risk in patients at high risk, but it may decrease the severity of the symptoms (32). Studies related with coasting are retrospective, and controlled randomized prospective studies do not exist. There is only one comprehensive study describing coasting protocol in literature that is the study of Mansour et al in 2005 (33). Pregnancy and implantation rates are reported to decrease when the duration of coasting exceeds four days $(33,34)$.

Standard (10.000 IU) and low-dose (5.000 IU) hCG administrations were compared in ovulation stimulation, and low-dose hCG was reported to be used successfully without damaging the clinical course (35), and to decrease the risk of OHSS (36). The problem with low-dose hCG stimulation is the increase in the number of cycle cancellations based on the decrease in oocyte retrieval (37). Furthermore, the number of controlled randomized studies in this respect is not sufficient. Determination of a dose threshold that decreases the risk for OHSS but does not prevent the oocyte maturation will increase the use of this method.

The long-term use of GnRH agonists causes down-regulation and desensitization of the receptors; however, their bolus administration in the stimulated cycles leads to pituitary FSH and LH releases similar to those seen in the natural cycle, which trigger ovulation. GnRHa administration for triggering ovulation minimizes OHSS risk, and does not cause a defect in the oocyte maturation. Oocyte pick-up is recommended in the $34^{\text {th }}$ to $35^{\text {th }}$ hours following the GnRHa administration (38). Trigger with GnRHa is only applied in the GnRH antagonist protocol, and it causes luteal phase insufficiency, leading to an increase in the early pregnancy losses (39).

Recombinant LH use for triggering ovulation, produces a pattern similar to the natural LH peak, and it is therefore recommended (40). It occurs with considerably high reliability in preventing OHSS, but it possesses low pregnancy rates, and cost/ benefit ratios limit the use of this protocol (41).
Agents like GnRH antagonists, dopamine agonists, IV albumin or HES (hydroxy ethyl starch) are being tested to save the cycles with increased E2 levels and high risk for OHSS. There is no data strong enough to prove that these agents decrease the OHSS risk, and this subject requires controlled randomized wide-spectrum studies (42).

Gürgan et al. (43) reported that intravenous calcium infusion might prevent the development of OHSS by blocking the renin-angiotensin system, and thus causing the VEGF levels to decrease.

In the randomized and controlled double-blind studies of El-Khayat and Elsadek (44). it was demonstrated that intravenous calcium treatment decreases the OHSS incidence without decreasing the pregnancy rates

\section{Oocyte-embryo qualities and deterioration of the fertilization rates}

A great number of oocytes are obtained in the IVF cycle stimulation of PCOS cases; however, oocyte and embryo qualities, fertilization rates, and cleavage and implantation rates are low; what is more, rate of early pregnancy losses is high, which is related with the increased risk of aneuploidy resulting from the damaged oocyte and embryo qualities (45). Damaged oocyte maturation and insufficient embryonic development in PCOS are related with the changes in intrafollicular microenvironment during the processes of folliculogenesis, follicular growth and meiotic oocyte maturation, abnormal endocrine and paracrine factors, and metabolic dysfunction. Clarification of the mechanisms of effects of these intraand extraovarian abnormalities in the granulosa-oocyte interaction, oocyte maturation, fertilization, embryonic development and pregnancy in PCOS cases will help to optimize the treatment protocols of these patients undergoing IVF, and to increase the pregnancy rates (46).

FSH determines the viability in folliculogenesis; the balance between the normal growth and atresia of the follicles is also maintained and determined by FSH. Antral follicles in the ovaries start to gain sensitivity to FSH when they are 2 $\mathrm{mm}$ to $5 \mathrm{~mm}$ in size, and slowly developing follicle gains aromatase activity when it is $6 \mathrm{~mm}$ to $8 \mathrm{~mm}$ in size, and thus gains the potential to increase E2 level (47). FSH levels drop in the late follicular phase with the simultaneous increases in E2 and inhibin. Finally, only one strong and fully matured follicle is selected for ovulation. At the end of the luteal phase, FSH increases slightly so that the subsequent ovulatory cycle can be initiated (48). FSH levels are lower in PCOS cases compared with the normal cycle, which results in the accumulation of antral follicles in $2 \mathrm{~mm}$ to $8 \mathrm{~mm}$ in sizes. The increased number of small follicles leads to premature arrest in most of them and the selection of the dominant follicle cannot be achieved (49). However in many studies, oocytes picked-up in PCOS cases were determined to be normal in development, and their ovaries were reported to have the po- 
tential for fertilization; the pregnancy rates were also similar to those of the normal ovaries (50).

E2 levels are high in the IVF cycles of the cases with PCOS. Oocytes picked-up in the PCOS IVF cycles were reported to be great in numbers, but high-quality oocytes were reported to be insufficient. These cycles occur with low blastocyst formation rates, and high embryonic fragmantation rates; they also possess low rates of fertilization and implantation (51). High E2 levels in the PCOS cases may lead to defects in oocyte maturation and embryonic development (52). Development of the immature oocytes with IVM application is a potential and useful choice of treatment in the PCOS infertility. Minimal or mild stimulation has also been applied with FSH before ovum pick-up as an alternative approach (53). Oocytes obtained immaturely due to low FSH levels will develop once the cumulus cell and oocyte interaction is achieved with extrinsic FSH (54). Although there are studies reporting the IVM results in PCOS comparable with those of the conventional IVF, current studies have demonstrated that IVM causes damage in the oocyte chromosomal configuration in the PCOS (55).

Tonic hypersecretion of LH occurs in the follicular phase in the natural cycles of the PCOS women. Hypersecretion of $\mathrm{LH}$ in the folliculogenesis induces atresia of the small antral follicles and the premature luteinization of the granulosa cells, leading to disordered granulosa cell functions, which results in the suppression of the oocyte maturation inhibitors, and thus causes premature maturation of the oocytes. Oocyte maturation disordered by the high LH in this way, results in low fertilization rate, low-quality embryos, followed by low pregnancy rates and high rates of early losses $(51,56)$. In addition, $\mathrm{LH}$ activates premature meiotic development by damaging the oocyte nucleus, and disordered endocrine control of the meiosis also damages the first polar body extrusion, which results in the final damage of the oocyte chromosomal configuration. This mechanism was also shown to play role in the development of aneuploidy in the PCOS $(45,57)$.

Androgen also increases in the follicular fluid in the PCOS cases, as well as in the circulation, which is related with the high LH levels, and it blocks follicular development, leading to follicular arrest and degeneration (56). The mechanism of testosterone activity in the oocyte is the exertion of negative effects on the meiotic maturation because of decreased calcium release, and inhibition of the oocyte cytoplasmic maturation (58). Damaged folliculogenesis and development of endometrial dysfunction because of the increased androgen level in PCOS are reported to cause increased early pregnancy losses (56).

Insulin resistance and compansatory hyperinsulinemia in PCOS may lead to damaged oocyte development, decreased rates of fertilization, embryonic development and implantation, and increased rates of pregnancy losses (51). Insulin may cause oocyte postmaturation and thus may damage its quality by increasing local androgen levels $(51,59)$. There are studies demonstrating that insulin-sensitizing agents decrease the rate of pregnancy losses in PCOS by decreasing the insulin levels (60). Defective glucose metabolism in the systemic level also exists in the follicular and ovarian levels in PCOS. It is considered that follicle and oocyte energy metabolisms are correlated with the oocyte maturation and embryonic quality (61). Difficulties in glucose transport into the oocyte and follicular cells, due to the defects in the glucose transport systems, induce the alternative energy pathways, which utilize free fatty acids, amino acids and ketone bodies (62). These compensating mechanisms lead to the different biomolecules in the follicular fluid to occur in altered levels. Follicular fluid is composed of plasma components that cross the follicular barrier, and it is modified by the secretory activity of the granulosa and theca cells. Changes in serum metabolites and the selective fitler activity of the granulosa cells play role in the metabolic changes in the follicular fluid. Follicular fluid includes important metabolites for the oocyte growth and development, and it reflects oocyte quality and embryo viability. Therefore, sufficient compensation maintains a high oocyte and embryo quality (63).

Intraovarian and extraovarian factors function in association with each other in the folliculogenesis. In oogenesis, the effects of intraovarian factors especially existing in the follicular fluid are predominant. Disrupted balance in these extraovarian and intraovarian factors in the PCOS leads to developmental insufficiency in the follicle and oocyte, and thus affects fertilization and embryonic development negatively (46).

EGF (epidermal growth factor) exists in the follicular fluid in the normal oogenesis, and it stimulates cumulus expansion and facilitates cytoplasmic maturation (64). However, a negative correlation exists between the oocyte and embryo qualities and increased follicular fluid EGF levels (65). Follicular fluid EGF levels are higher than normal in cases with PCOS, and EGF blocks antral follicular growth and causes follicular arrest by inhibiting estrogen synthesis in the granulosa cells in PCOS (66).

IGF (insulin-like growth factor) I-II and IGFBP (IGFbinding protein) possess important regulatory functions in the ovarian follicular development. There are various studies related with the increased insulin concentration and changes of IGF expression in PCOS; however, the role of IGFs in the pathogenesis of PCOS has not yet been completely clarified. Follicular fluid IGF-1 level was found to be high, and IGF-II and IGFBP-1 levels were found to be low in the PCOS, and the low IGFBP-1 was identified as being related with the follicular arrest in PCOS (66). IGF1, II and IGFBP-3 levels in the mature follicles in PCOS were shown to be similar with those of the normoovulatory women, but decreased IGF-1 level in the immature follicles during ovulation induction was found to be related with the follicular maturation block (67). 
Ongoing studies suggest the relations of FGF (fibroblast growth factor) and NGF (neurotrophin growth factor) with the oocyte and embryonic sufficiency; however, their roles in the pathogenesis of PCOS, and the level of blockade they cause have not yet been exactly ascertained $(66,68)$.

The TGF-B (transforming growth factor-beta) family includes members that have important biological roles in the folliculogenesis and oogenesis, as follows: AMH (anti mullerian hormone), activin, inhibins, follistatin, BMP-9 (bone morphogenic protein), and GDF-9 (growth differentiation factor). TGF-B family members may play role in the PCOS pathogenesis by inducing or blocking the follicular growth and/or granulosa-oocyte complex differentiation, in different physiological conditions $(46,56)$. High AMH levels in the serum and follicular fluid were found to be closely related with the increased antral follicle development and arrest of follicular development in PCOS (69). Increased AMH levels were shown to be directly correlated with the increased androgen and LH levels, and to cause disruption of the oocyte developmental sufficiency and embryonic quality (70). In another study, increased AMH was shown to disrupt oocyte quality and maturation by an unknown mechanism, but not to affect pregnancy rates (71). On the contrary, it was reported in the same period in another study that the rates of fertilization, implantation, and clinical pregnancy were higher in the group with high AMH level (72). It is recommended to use serum and follicular fluid AMH levels only in the predictivity of OHSS, but not in the predictivity of pregnancy in the IVF applications (73).

VEGF (vascular endothelial growth factor) plays important roles in the follicular vascularization, intrafollicular oxygenation, and thus the follicular maturation, oocyte quality, fertilization, and the sufficient embryonic development (74). Serum and follicular fluid VEGF levels in the normoovulatory women are correlated with a good ovarian response, increase in the oocyte retrieval and the improved fertilization and pregnancy rates; being contrary, elevated follicular fluid and serum VEGF levels are associated with a poor oocyte quality and decreased pregnancy rates especially at advanced ages. Increased follicular fluid VEGF levels in PCOS were found to be closely correlated with the development of OHSS, and high VEGF levels were shown to indicate immature oocyte and disordered fertilization (75). However the opposite results were obtained in another study, and increased follicular fluid VEGF was determined to affect the oocyte quality positively, and to increase retrieval of the MII oocytes (76). A study group demonstrated increased follicular fluid VEGF in the ovaries exposed to prolonged hCG effect in the PCOS patients, and they also showed increased oocyte and embryonic qualities and improved fertilization rates (77); after a couple of years, the same group showed decrease of VEGF and E2 levels in the follicular fluid in the PCOS cases following the administration of $\mathrm{GnRH}$ antagonist, and they determined that this resulted in disordered follicular develop- ment, and decreased oocyte and embryonic qualities, compared to the agonist-administered cycles (78).

A wide-spectrum of cytokine family consisting of such factors as IL (interleukins), LIF (leukemia inhibitory factor), TNF (tumor necrosis factor)-alpha, sFas (soluble Fas) and sFasL (sFas ligand) may exert autocrine or paracrine effects on the ovary, but they do not have endocrine effects (79). Their presence in the follicular fluid indicates their production in the granulosa cells, and they were shown to have roles in the follicular maturation and the following embryonic development (80). Cytokines were demonstrated to play a role in the OHSS (81) and hyperandrogenism (82) in the PCOS cases; however, controversial results were also obtained (83). Increased interleukin and TNF values in the follicular fluid were shown to be related with the disrupted oocyte maturation, decreased fertilization rates and decreased embryonic quality $(79,83)$.

Multiple endocrine and metabolic disorders in PCOS may potentially damage folliculogenesis, and oocyte maturation; when the oocytes of the PCOS patients and normal individuals were compared, various differences in their contents were also observed. MII oocytes recruited from the PCOS cases possess changes in the meiosis- and mitosis-related genes, genes related with the androgen receptor-binding site, and the expression of the genes related with the selection of the primary follicle (84). Despite all these findings, there are also meta-analyses demonstrating similar pregnancy rates in IVF in patients with PCOS and in cases with normal ovaries (50); this shows the ability of the PCOS patients to produce highquality oocytes. The general low-quality oocytes in PCOS cases may be compensated by the increased number of embryos obtained.

Oocyte quality is a fundamental requirement for embryonic development. In a study investigating the effect of PCOS on the kinetics of early embryonic cleavage, using the timelapse visualization method (85), embryos obtained from the hyperandrogenic PCOS cases were shown to develop at slower rates from fertilization to the 8-cell stage, when compared with the control group; no difference was detected in the normo-androgenic PCOS cases. Mitochondria of the preimplantation embryo is only of maternal origin, since paternal mitochondria is non-viable after the fertilization (86). Therefore, the mitochondrial dysfunction in the oocyte was considered to be responsible for the slowly developing early embryo in the hyperandrogenic PCOS cases. The clinical significance of this developmental difference is not yet known, since fertilization and pregnancy rates were not determined in this study.

Treatment strategies for improving the oocyte and embryo qualities in PCOS are still being widely studied and discussed. The addition of myo-inositol and chiro-inositol to the treatment was shown to increase oocyte and embryo qualities with 
the regulation of insulin metabolism (87). However, when used in the PCOS cases with a lack of insulin resistance, inositol may damage ovarian response and oocyte quality in a dose-dependent manner (88), and do not increase the rates of clinical pregnancy (89); this subject is still under discussion (90). The use of L-carnitine was shown to increase lipid consumption in the follicle, and oocyte quality was improved (91). Electroacupuncture may increase the oocyte and embryo qualities in the PCOS by increasing the levels of stem cell factor (92). Cytoplasmic maturation and the following fertilization and implantation rates may improve by adding melatonin, an endogenous antioxidant, into the in vitro culture medium in PCOS (93).

Autologous cumulus cell co-culture studies are promising in increasing the oocyte and embryo qualities in the PCOS. It has been determined that ratio of grade I embryos and blastulation rates increased although oocyte retrieval and ratio of MII oocytes did not differ, and the rates of fertilization and clinical pregnancy also increased nonsignificantly in the PCOS co-culture group, compared to the control group (94).

\section{Deterioration of the endometrial receptivity and implantation rates}

Implantation is a priority for the maintenance of a successful pregnancy. Embryo quality is an important indicator of the implantation; however, transient differentiation of the uterine cells to maintain uterine receptivity and synchronization between the maternal and embryonic tissues are also fundamental requirements (95).

The major factor responsible for the infertility in PCOS is considered to be the chronic anovulation; however, an increasing number of data support the role of unsuccessful implantation in the PCOS infertility. Anovulation is problem that may be dealt with by medical induction; however, implantation rates are low and rates of early pregnancy losses are high in the PCOS when compared with the fertile control groups (96).

Progesterone resistance exists in the endometria of the PCOS cases at the level of gene expression; regulatory effect of progesterone on the endometrium is insufficient or it is absent, and estrogen response is increased. This atypical decidualization, developing as a result of abnormal estrogen and progesterone interaction, is one of the reasons for PCOS subfertility (97).

Steroid receptors and their coactivators also differ in women with PCOS, compared with fertile controls. The expression of androgen receptors in the PCOS endometrium is excessive, and down-regulation of the estrogen receptors is insufficient during the implantation period (98). What is more, estrogen activity of the endometrial cells in women with PCOS is marked by the excessive expression of the steroid receptor coactivators AIB1 (nuclear receptor coactivator 3) and TIF2 (transcriptional intermediary factor 2) (99).
Various studies have shown that disorders in the expression of the uterine receptivity markers (integrin, selectin, cadherin, glycodelin, osteopontin, mucin, HOXA 10, IGFBP-1) exist in the PCOS. Expressions of the alphaVB3 integrin, HOXA 10 and IGFBP-1 were shown to decrease in the secretory phase in the ovulatory PCOS cases (100). In a study reviewing the results of the last ten years related to the adhesion molecules and estrogen receptors, it was reported that the cell adhesion molecule MUC1 (mucin) expression is high and the alphaVB3 integrin expression is low, and also that expression of estrogen receptor is increased in the PCOS cases compared with healthy women. These results have led to the conclusion that disordered regulation of these endometrial molecular mediators causes disordered endometrial receptivity, and thus disrupted embryo implantation (101). Testosterone levels were shown to decrease by HOXA-10 expression in in vitro conditions, and androgen decrease was considered to improve the endometrial receptivity. When the insulin level was decreased with metformin, serum glycodelin and IGFBP1 levels were demonstrated to decrease and uterine vascularity and blood flow were shown to increase in the luteal phase (102).

Endometrium is a target tissue for insulin, which acts during the secretory phase via the insulin receptors that increase with the effect of progesterone, or via the IGF receptors, which are similar in structure (103). The IGF system, which is composed of the IGF-1 and II, IGF receptors and IGFBPs, is mitogenic for the endometrial cells, and it also regulates the secretory functions of the stromal cells. They contribute to the endometrial proliferation and development, and the implantation (104). In in vitro conditions, insulin inhibits the endometrial stromal production of the IGFBP-1, which is a decidualization marker (105). It has been reported that this inhibition is achieved via the PI3 (phosphatidyl inositol-3) kinase pathway, mediated by the low insulin levels; in the presence of high insulin doses, and MAPK (mitogen activated protein kinase) pathway is activated. These observations have led to the conclusions that insulin in physiological levels play a homeostatic role in the endometrial energy metabolism, and that hyperinsulinemic status activates the cellular mitosis via the MAPK pathway, thus leading to endometrial hyperplasia and tendency for cancer. It is considerably difficult in in vivo conditions to discriminate the effects of insulin on endometrium, from those of androgen, since hyperandrogenism and hyperinsulinemia correlate positively in the anovulatory state (106). Metabolic changes such as the glucose metabolism, which regulates decidualization, hyperinsulinemia, and hyperandrogenism in the obesity of PCOS cases, were shown to cause disordered endometrial receptivity during implantation (107).

Changes in the endometrial leukocyte subpopulations, and especially in the NK-cell ratios in the secretory phase of the normal menstrual cycle, are steroid hormone-dependent, and play important roles in the implantation and maintenance of the pregnancy (96). Uterine NK-cells contribute to the prepa- 
ration of a local immunosuppressive microenvironment for implantation, by leading to expression of immunomodulatory molecules. In the infertile PCOS group, disordered cytokine balance in the secretory endometrium was shown to be associated with the disordered endometrial lymphocyte pattern, and especially disordered natural killer cell ratios. The disordered expression of some receptivity markers such as glycodelin-A, which is expressed selectively by the uterine NK cells in the secretory phase endometrium of the patients with PCOS, was investigated (108). Endometrial immune system defect during implantation contributes to the low reproductive capacity existing in the patients with PCOS (109).

Hypercoagulability existing during the peri-implantation phase in PCOS was considered to be one of the factors that mostly affecting implantation success in PCOS, by causing disordered endometrial microcirculation (110).

The effects of high E2 levels on embryonic implantation in the stimulated cycle in PCOS are a matter of debate. Moreover, in addition to the studies showing disrupted endometrial receptivity and implantation by the elevated E2 levels (111), there are also studies demonstrating that high E2 levels do not negatively affect the IVF results (112). A further study has demonstrated that increased E2 levels exert a direct toxic effect on the embryo during cleavage; in this study, late embryo transfer was considered to minimize the negative effects of high E2 levels, by shortening the duration that the embryo is exposed to high E2 during cleavage (113).

Rates of early pregnancy losses and repetitive pregnancy losses are high in PCOS. In the patients without PCOS, but with the history of unexplained and repetitive pregnancy losses, high circulatory LH and free testosterone levels, low luteal progesterone levels, and a slowly developing endometrium were observed (96). Endometrial receptivity markers were also found to be low in these cases (100). Most of these findings occur in those patients with PCOS that are treated for infertility. Patients with high androgen levels were shown to have higher rates of unsuccessful implantation and early pregnancy losses, compared with the patients in the same age interval, and to have similar weights and insulin levels (114). In light of these data, it must be kept in mind that patients with PCOS are also at increased risk of early pregnancy loss, and in the management of infertility, approaches have to be planned to take this fact into consideration.

As a conclusion, patients with PCOS constitute the most difficult population in the management of infertility. The factors that increase the success rates in the treatment of PCOS infertility are: pretreatment changes in life style, dietetic and psychological support, a detailed evaluation of the couple and the appropriate selection of the treatment protocol, a widespectrum approach to maintaining ovarian and endometrial synchronization in the management of the cycle, and well-developed laboratory conditions to support embryonic quality.
However, even if these conditions are provided, OHSS, cancellations of the cycle, poor oocyte, and embryo qualities, unsuccessful fertilization and implantation, chromosomal abnormalities and early losses may still be experienced. In order to overcome this problem of infertility, an experienced team with a rigorous knowledge of the problem, supported by a high level of technology is required. Tolerant collaboration from this team and a cooperative couple are essential.

\section{References}

1. Fauser BCJM. Tarlatzis BC. Rebar RW. Legro RS. Balen AH. Lobo R. \& Barnhart K. Consensus on women's health aspects of polycystic ovary syndrome (PCOS). Human Reproduction 2012;27;(1):14-24.

2. Dunaif A. and Fauser BC. Renaming PCOS-a two-state solution. The Journal of Clinical Endocrinology \& Metabolism 2013;98(11):4325-8.

3. The Rotterdam ESHRE/ASRM-Sponsored PCOS Consensus Workshop Group. Revised 2003 consensus on diagnostic criteria and long-term health risks related to polycystic ovary syndrome. Fertil Steril 2004;81:19-25.

4. Eshre TT. Group, ASPCW. Consensus on infertility treatment related to polycystic ovary syndrome. Fertility and sterility 2008;89(3):505-22.

5. MacDougall MJ, Tan SL, et al. A controlled study comparing patients with and without polycystic ovaries undergoing in-vitro fertilization. Hum Reprod 1993;8:233-7

6. Lyons CAD. Wheeler CA. Frishman GN. Hackett RJ. Seifer DB. Haning RV. Early and late presentation of the ovarian hyperstimulation syndrome: two distinct entities with different risk factors.Human Reproduction 1994; 9(5):792-9.

7. Humaidan P. Quartarolo J. Papanikolaou EG. Preventing ovarian hyperstimulation syndrome: guidance for the clinician. Fertility and sterility 2010;94(2):389-400.

8. Shulman A, Dor J. In vitro fertilization treatment in patients with polycystic ovaries. J Assist Reprod Genet 1997;14:7-10.

9. Kamat BR, Brown LF, Manseau EJ, Senger DR, Dvorak HF. Expression of vascular permeability factor/vascular endothelial growth factor by human granulosa and theca lutein cells. Role in corpus luteum development. Am J Pathol 1995;146:157-65.

10. Danninger B, Brunner M, Obruca A, Feichtinger W. Prediction of ovarian hyperstimulation syndrome by ultrasound volumetric assessment [corrected] of baseline ovarian volume prior to stimulation. Hum Reprod 1996;11: 1597-9.

11. Moohan JM, Curcio K, Leoni M, Healy D, Hurley V. Low intraovarian vascular resistance: a marker for severe ovarian hyperstimulation syndrome. Fertil Steril 1997;67:728-32. 
12. Bodis J, Torok A, Tinneberg HR. LH/FSH ratio as a predictor of ovarian hyperstimulation syndrome. Hum Reprod 1997;12:869-70.

13. Lee TH, Liu CH, Huang CC, Wu YL, Shih YT, Ho HN, et al. Serum anti-mullerian hormone and estradiol levels as predictors of ovarian hyperstimulation syndrome in assisted reproduction technology cycles. Hum Reprod 2008;23:160-7.

14. Papanikolaou EG. Humaidan P. Polyzos N. Kalantaridou S. Kol S. Benadiva C. Tarlatzis B. New algorithm for OHSS prevention. Reprod Biol Endocrinol 2011;9(1):147.

15. Kligman I. Rosenwaks Z. Differentiating clinical profiles: predicting good responders, poor responders, and hyperresponders. Fertil steril 2001;76(6):1185-90.

16. Papanikolaou EG, Pozzobon C, Kolibianakis EM, Camus M, Tournaye H, Fatemi HM, et al. Incidence and prediction of ovarian hyperstimulation syndrome in women undergoing gonadotropinreleasing hormone antagonist in vitro fertilization cycles. Fertil Steril 2006;85:112-20.

17. Pau E, Alonso-Muriel I, Gomez R, Novella E, Ruiz A, Garcia-Velasco JA, et al. Plasma levels of soluble vascular endothelial growth factor receptor-1 may determine the onset of early and late ovarian hyperstimulation syndrome. Hum Reprod 2006;21:1453-60.

18. Al-Azemi M. Killick SR. Duffy S. Pye C. Refaat B. Hill, N. Ledger W. Multi-marker assessment of ovarian reserve predicts oocyte yield after ovulation induction. Hum reprod 2011;26(2):414-22.

19. Kolibianakis EM, Albano C, Camus M, Tournaye H, Van Steirteghem AC, Devroey P. Prolongation of the follicular phase in in vitro fertilization results in a lower ongoing pregnancy rate in cycles stimulated with recombinant follicle-stimulating hormone and gonadotropin-releasing hormone antagonists. Fertil Steril 2004;82:102-7.

20. Pelinck MJ, Vogel NEA, Hoek A, Arts EGJM, Simons AHM, Heineman MJ. Minimal stimulation IVF with late follicular phase administration of the GnRH antagonist cetrorelix and concomitant substitution with recombinant FSH: a pilot study. Hum Reprod 2005;20:642-8

21. Al-Inany HG, Abou-Setta AM, Aboulghar M. Gonadotrophin - releasing hormone antagonists for assisted conception. Cochrane Database Syst Rev 2006;(3):CD001750.

22. Kolibianakis EM, Collins J, Tarlatzis BC, Devroey P, Diedrich K, Griesinger G. Among patients treated for IVF with gonadotrophins and GnRH analogues, is the probability of live birth dependent on the type of analogue used? A systematic review and meta-analysis. Hum Reprod Update 2006;12(2):651-71

23. Check JH. Luteal phase support for in vitro fertilizationembryo transfer-present and future methods to improve successful implantation. Clinical and Experimental Obstetrics \& Gynecology 2011;39(4):422-8.

24. Daya S, Gunby J. Luteal phase support in assisted repro- duction cycles. Cochrane Database Syst Rev 2004;(3): CD004830

25. Pirard C, Donnez J, Loumaye E. GnRH agonist as novel luteal support: results of a randomized, parallel group, feasibility study using intranasal administration of buserelin. Human Reprod 2005;20:1798-804.

26. Grameau A, Andreadis N, Fatum M, Craig J, Turner K, McVeigh E, Child T. In vitro maturation of in vitro fertilization for women with polycystic ovaries? A case control study of 194 treatment cycles. Fertil Steril 2012;98:35560 .

27. Child TJ, Phillips SJ, Abdl-Jalil AK, Guleki B, Tan SL. A comparison of in vitro maturation and in vitro fertilization for women with polycystic ovaries. Obstet Gynecol 2002;100:665-70.

28. Le Du A, Kadoch IJ, Bourcigaux N, Doumerc S, Bourrier $\mathrm{MC}, \mathrm{Chevalier} \mathrm{N}$, et al. In vitro oocyte maturation for the treatment of infertility associated with polycystic ovarian syndrome:the French experience. Hum Reprod 2005;20: 420-4

29. Schenker JG, Weinstein D. Ovarian hyperstimulation syndrome: a current survey. Fertil Steril 1978;30:255-68.

30. Shapiro BS. Daneshmand ST. Garner FC. Aguirre M. Hudson C. Thomas S. Evidence of impaired endometrial receptivity after ovarian stimulation for in vitro fertilization: a prospective randomized trial comparing fresh and frozen-thawed embryo transfer in normal responders. Fertil Steril 2011;96(2):344-8.

31. Tozer AJ. Iles RK. Iammarrone E. Gillott CM. Al-Shawaf T. Grudzinskas JG. The Effects of "Coasting" on Follicular Fluid Concentrations of Vascular Endothelial Growth Factor in Women at Risk of Developing Ovarian Hyperstimulation Syndrome. Hum Reprod 2004;19(3): $522-8$

32. Delvigne A, Rozenberg SA Qualitative Systematic Review of Coasting, a Procedure to Avoid Ovarian Hyperstimulation Syndrome in IVF Patients. Hum Reprod Update 2002;8(3):291-6

33. Mansour R, Aboulghar M, Serour G, Amin Y, Abou-Setta AM. Criteria of a Successful Coasting Protocol for the Prevention of Severe Ovarian Hyperstimulation Syndrome. Hum Reprod 2005;20(11):3167-72.

34. Levinsohn-Tavor O, Friedler S, Schachter M, Raziel A, Strassburger D, Ron-El R. Coasting - What Is the Best Formula? Hum Reprod 2003;18(5):937-940.

35. Schmidt DW, Maier DB, Nulsen JC, Benadiva CA. Reducing the dose of human chorionic gonadotropin in high responders does not affect the outcomes of in vitro fertilization. Fertil Steril 2004;82:841-6.

36. Kashyap S, Leveille M, Wells G. Low dose hCG reduces the incidence of early and severe ovarian hyperstimulation syndrome. Fertil Steril 2006;86(Suppl 2):182-3 (P-138).

37. Abdalla HI, Ah-Moye M, Brinsden P, Howe DL, 
Okonofua F, Craft I. The effect of the dose of human chorionic gonadotropin and the type of gonadotropin stimulation on oocyte recovery rates in an in vitro fertilization program. Fertil Steril 1987;48:958-63.

38. Humaidan P. Kol S. Papanikolaou EG. on behalf of the "The Copenhagen GnRH Agonist Triggering Workshop Group: GnRH agonist for triggering offinal oocyte maturation: time for a change of practice? Hum Reprod Update 2011;17:510-524.

39. Engmann L. DiLuigi A. Schmidt D. Nulsen J. Maier D. Benadiva $\mathrm{C}$. The use of gonadotropin-releasing hormone $(\mathrm{GnRH})$ agonist to induce oocyte maturation after cotreatment with GnRH antagonist in high-risk patients undergoing in vitro fertilization prevents the risk of ovarian hyperstimulation syndrome: a prospective randomized controlled study. Fertil Steril 2008;89:84-91.

40. Emperaire JC, Edwards RG. Time to revolutionize the triggering of ovulation. Reprod Biomed Online 2004; 9:480-3

41. European Recombinant LH Study Group. Human Recombinant Luteinizing Hormone Is as Effective as, But Safer Than, Urinary Human Chorionic Gonadotropin in Inducing Final Follicular Maturation and Ovulation in in Vitro Fertilization Procedures: Results of a Multicenter Double-Blind Study 1. The J Clin Endocri \& Metabolism 2001;86(6);2607-18.

42. Fiedler K. Ezcurra D. Predicting and preventing ovarian hyperstimulation syndrome (OHSS): the need for individualized not standardized treatment. Reprod Biol Endocrinol 2012;10(1):32-41.

43. Gurgan T. Demirol A. Guven S. Benkhalifa M. Girgin B. Li TC. Intravenous calcium infusion as a novel preventive therapy of ovarian hyperstimulation syndrome for patients with polycystic ovarian syndrome. Fertil Steril 2011;96 (1):53-7.

44. El-Khayat W. Elsadek M. Calcium infusion for the prevention of ovarian hyperstimulation syndrome: a doubleblind randomized controlled trial.Fertility and sterility 2015;103(1):101-5.

45. Weghofer A, Munne S, Chen S, Barad D, Gleicher N. Lack of association between polycystic ovary syndrome and embryonic aneuploidy. Fertil Steril 2007;88:900-905.

46. Qiao J. Feng HL. Extra-and intra-ovarian factors in polycystic ovary syndrome: impact on oocyte maturation and embryo developmental competence. Hum Reprod update, dmq032. 2010.

47. Dumesic DA.\&Abbott DH. Implications of Polycystic Ovary Syndrome (PCOS) on Oocyte Development. In Seminars in Reprod Med 2008;26:53. NIH Public Access.

48. Padhy N. Latha M. Sathya B. Varma TR. Antral follicle size in the downregulated cycle and its relation to in vitro fertilization outcome. J Human Rep sciences 2009;2(2):68.

49. Hillier SG. Current concepts of the roles of follicle stimu- lating hormone and luteinizing hormone in folliculogenesis. Hum Reprod 1994;9(2):188-91.

50. Heijnen EMEW. Eijkemans MJC. Hughes EG. Laven JS. E. Macklon N. Fauser BCJM. A meta-analysis of outcomes of conventional IVF in women with polycystic ovary syndrome. Hum reprod update 2006;12(1):13-21.

51. Cano F, Garcia-Velasco JA, Millet A, Remohi J, Simon C, Pellicer A. Oocyte quality in polycystic ovaries revisited: identification of a particular subgroup of women. J Assist Reprod Genet 1997;14:254-60.

52. Hardy K. Robinson FM. Paraschos T. Wicks R. Franks S. Winston RM. Fertilization and early embryology: Normal development and metabolic activity of preimplantation embryos in vitro from patients with polycystic ovaries. Hum Reprod 1995;10(8):2125-35.

53. Chian RC. Buckett WM. Tulandi T. Tan SL. Prospective randomized study of human chorionic gonadotrophin priming before immature oocyte retrieval from unstimulated women with polycystic ovarian syndrome. Hum Reprod 2000;15(1):165-70.

54. Dumesic DA. Padmanabhan V. Abbott DH. Polycystic ovary syndrome and oocyte developmental competence. Obstet \& Gynecol Survey 2008;63(1):39.

55. Nichols SM. Gierbolini L. Gonzalez-Martinez JA. Bavister BD. Effects of in vitro maturation and age on oocyte quality in the rhesus macaque Macaca mulatta. Fertil Steril 2010;93(5):1591-1600.

56. van der Spuy ZM. Dyer SJ. The pathogenesis of infertility and early pregnancy loss in polycystic ovary syndrome. Best Practice \& Research Clinical Obstetrics \& Gynaecology 2004;18(5):755-71.

57. Weghofer A. Munné S. Brannath W. Chen S. Tomkin G. Cekleniak N. Gleicher N. The impact of LH-containing gonadotropins on diploidy rates in preimplantation embryos: long protocol stimulation. Human Reproduction 2008;23(3): 499-503.

58. Tesarik J. Mendoza C. Direct non-genomic effects of follicular steroids on maturing human oocytes: oestrogen versus androgen antagonism.Human reproduction update 1997;3(2):95-100.

59. Cano F. Velasco JAG. Millet A. Remohi J. Simon C.Pellicer A. Oocyte quality in polycystic ovaries revisited: Identification of a particular subgroup of women. J Assist Reprod Genet 1997;14:254-261

60. Tang T. Lord J. M. Norman R J. Yasmin E. Balen AH. Insulin-sensitising drugs (metformin, rosiglitazone, pioglitazone, D-chiro-inositol) for women with polycystic ovary syndrome, oligo amenorrhoea and subfertility. The Cochrane Library 2009.

61. Arya BK. Haq AU. Chaudhury K. Oocyte quality reflected by follicular fluid analysis in poly cystic ovary syndrome (PCOS): A hypothesis based on intermediates of energy metabolism. Medical Hypotheses 2012;78(4): 
$475-8$

62. Gull I. Geva E. Lerner-Geva L. Lessing JB. Wolman I. Amit A. Anaerobic glycolysis: the metabolism of the preovulatory human oocyte. Euro J Obstet Gynecol Reprod Bio 1999;85(2): 225-8.

63. Piñero-Sagredo E. Nunes S. de los Santos M. Celda, B. Esteve V. NMR metabolic profile of human follicular fluid. NMR in Biomedicine 2010;23(5):485-95.

64. Goff AK. Yang Z. Cortvrindt R. Smitz J. Miron P. Protein synthesis during maturation of bovine oocytes, effect of epidermal growth factor. Reproduction in Domestic Animals 2001;36(1):19-24.

65. Hofmann GE. Scott Jr, RT. Brzyski RG. Jones Jr, HW. Immunoreactive epidermal growth factor concentrations in follicular fluid obtained from in vitro fertilization. Fertil Steril 1990;54(2):303-7.

66. Artini AP. Monteleone P. Parisen Toldin MR. Matteucci C. Ruggiero M. Cela V. Genazzani AR. Growth factors and folliculogenesis in polycystic ovary patients Expert Revi Endoc Metabol 2007;2(2):215-23

67. Schoyer KD. Liu HC. Witkin S. Rosenwaks Z. Spandorfer SD. Serum insulin-like growth factor I (IGF-I) and IGFbinding protein 3 (IGFBP-3) in IVF patients with polycystic ovary syndrome: correlations with outcome. Fertil Steril 2007;88(1):139-44.

68. Buyuk E. Seifer DB. Follicular-fluid neurotrophin levels in women undergoing assisted reproductive technology for different etiologies of infertility. Fertil Steril 2008;90(5):1611-5.

69. Pigny P. Merlen E. Robert Y. Cortet-Rudelli C. Decanter C. Jonard S. Dewailly D. Elevated serum level of antimullerian hormone in patients with polycystic ovary syndrome: relationship to the ovarian follicle excess and to the follicular arrest. J Clin Endoc Metabol 2003;88(12):5957-62.

70. Fallat ME. Siow Y. Marra M. Cook C. Carrillo A. Müllerian-inhibiting substance in follicular fluid and serum: a comparison of patients with tubal factor infertility, polycystic ovary syndrome, and endometriosis. Fertil Steril 1997;67(5):962-5.

71. Desforges-Bullet V. Gallo C. Lefebvre C. Pigny P. Dewailly D. Catteau-Jonard S. Increased anti-Müllerian hormone and decreased FSH levels in follicular fluid obtained in women with polycystic ovaries at the time of follicle puncture for in vitro fertilization. Fertil Steril 2010;94(1):198-204.

72. Pabuccu R. Kaya C. Cağlar GS. Oztas E. Satiroglu H. Follicular-fluid anti-Mullerian hormone concentrations are predictive of assisted reproduction outcome in PCOS patients. Reprod Biomed Online 2009;19(5):631-7.

73. Lin W. Q. Yao LN. Zhang DX. Zhang W. Yang XJ. Yu R. The predictive value of anti-mullerian hormone on embryo quality, blastocyst development, and pregnancy rate following in vitro fertilization-embryo transfer (IVF-ET). J Assis Reprod Genet 2013;30(5): 649-55.

74. Agrawal R. Conway G. Sladkevicius P. Tan SL. Engmann, L. Payne N. Jacobs H. Serum vascular endothelial growth factor and Doppler blood flow velocities in in vitro fertilization: relevance to ovarian hyperstimulation syndrome and polycystic ovaries. Fertil Steril 1998;70(4):651-8.

75. Artini PG. Monti M. Matteucci C. Valentino V. Cristello F. Genazzani AR. Vascular endothelial growth factor and basic fibroblast growth factor in polycystic ovary syndrome during controlled ovarian hyperstimulation. Gynecol Endocrin 2006;22(8):465-70.

76. Artini PG. Ruggiero M. Parisen Toldin MR. Monteleone P. Monti M. Cela V. Genazzani AR. Vascular endothelial growth factor and its soluble receptor in patients with polycystic ovary syndrome undergoing IVF. Hum Fertil 2009;12(1):40-4.

77. Vrtačnik-Bokal EV. Vrtovec HM. Klun IV. Verdenik I. Prolonged HCG action affects angiogenic substances and improves follicular maturation, oocyte quality and fertilization competence in patients with polycystic ovarian syndrome. Human Reprod 2005;20(6):1562-8

78. Vrtačnik-Bokal E. Klun IV. Verdenik I. Follicular oestradiol and VEGF after GnRH antagonists or GnRH agonists in women with PCOS. Reprod Biomed Online 2009; 18(1):21-8.

79. Zolti M. Bider D. Seidman DS. Mashiach S. Ben-Rafael ZION. Cytokine levels in follicular fluid of polycystic ovaries in patients treated with dexamethasone. Int $\mathrm{J}$ Gynecol Obstet 1992; 39(3):256-7.

80. Wu R. Fujii S. Ryan NK. Van der Hoek KH. Jasper MJ. Sini I. Norman RJ. Ovarian leukocyte distribution and cytokine/chemokine mRNA expression in follicular fluid cells in women with polycystic ovary syndrome. Hum Reprod 2007;22(2):527-35.

81. Pellicer A. Albert C. Mercader A. Bonilla-Musoles F. Remohí J. Simón C. The pathogenesis of ovarian hyperstimulation syndrome: in vivo studies investigating the role of interleukin-1 $\beta$, interleukin-6, and vascular endothelial growth factor. Fertil Steril 1999;71(3): 482-9.

82. Escobar-Morreale HF. Calvo RM. Sancho J. San Millán JL. TNF- $\alpha$ and hyperandrogenism: a clinical, biochemical, and molecular genetic study. J Clin Endocrin Metabol 2001;86(8):3761-7.

83. Amato G. Conte M. Mazziotti G. Lalli E. Vitolo G. Tucker AT. Izzo A. Serum and follicular fluid cytokines in polycystic ovary syndrome during stimulated cycles. Obstet Gynecol 2003;101(6):1177-82.

84. Wood JR. Dumesic DA. Abbott DH. Strauss III, JF. Molecular abnormalities in oocytes from women with polycystic ovary syndrome revealed by microarray analysis. J Clin Endoc Metabol 2007;92(2):705-13.

85. Wissing ML. Bjerge MR. Olesen AI. G. Hoest T. 
Mikkelsen AL. Impact of PCOS on early embryo cleavage kinetics. Reprod Biomed Online 2014;28(4):508-14.

86. Craigen WJ. Mitochondrial DNA mutations: an overview of clinical and molecular aspects. Methods Mol Biol $2012 ; 837: 3-15$

87. Unfer V. Carlomagno G. Papaleo, E. Vailati S. Candiani M. Baillargeon JP. Hyperinsulinemia alters myoinositol to D-chiroinositol ratio in the follicular fluid of patients with PCOS. Reproductive Sciences 2014;193371911351 8985.

88. Isabella R. \& Raffone E. Does ovary need D-chiro-inositol. J Ovarian Res 2012;5(1):14.

89. Lisi F. Carfagna P. Oliva MM. Rago R. Lisi R. Poverini R. Moscarini M. Pretreatment with myo-inositol in non polycystic ovary syndrome patients undergoing multiple follicular stimulation for IVF: a pilot study. Reprod Biol Endocl 2012;10:52.

90. Vitagliano A. Quaranta M. Noventa M. Gizzo S. "Empiric" inositol supplementation in normal-weight non insulin resistant women with polycystic ovarian disease: from the absence of benefit to the potential adverse effects. Arch Gynecol Obstet 2015;1-3.

91. Dunning KR. Robker RL. Promoting lipid utilization with L-carnitine to improve oocyte quality. Animal Reprod Science 2012;134 (1-2):69-75.

92. Cui W. Li J. Sun W. Wen J. Effect of electroacupuncture on oocyte quality and pregnancy of patients with PCOS undergoing in vitro fertilization and embryo transfer. World J Acupuncture-Moxibustion 2012;22(1):23-9.

93. Kim MK. Park EA. Kim HJ. Choi WY. Cho JH. Lee WS. Yoon TK. Does supplementation of in-vitro culture medium with melatonin improve IVF outcome in PCOS? Reprod Biomed Online 2013;26(1):22-9.

94. Demirol A. Guven S. Benkhalifa M. Girgin B. Gurgan T. Hiraoka K. Yoshida A. Embryology-Laboratory Procedures (Session 38). Hum Reprod 2012;27(suppl 2):56-7.

95. C. Simón JC. Martín A. Pellicer. Paracrine regulators of implantation. Bailliere. Best Pract Res Clin Obstet Gynaecol 2000;14:815-26

96. Giudice LC. Endometrium in PCOS: implantation and predisposition to endocrine CA. Best Practic Res Clin Endoc Metabol 2006;20(2):235-44.

97. Piltonen TT. Chen J. Khatun M. Kangasniemi M. Liakka A. Spitzer T. Giudice LC. Endometrial stromal fibroblasts from women with polycystic ovary syndrome have impaired progesterone-mediated decidualization, aberrant cytokine profiles and promote enhanced immune cell migration in vitro. Hum Reprod 2015;30(5)

98. Apparao KBC. Lovely LP. Gui Y. Lininger RA. Lessey BA. Elevated endometrial androgen receptor expression in women with polycystic ovarian syndrome. Biol Reprod 2002;66(2):297-304.

99. Gregory CW. Wilson EM. Apparao KBC. Lininger RA.
Meyer WR. Kowalik A. Lessey BA. Steroid receptor coactivator expression throughout the menstrual cycle in normal and abnormal endometrium. J Clin Endoc Metabol 2002;87(6):2960-6.

100. Cakmak H. Taylor HS. Implantation failure: molecular mechanisms and clinical treatment. Hum Reprod Update 2011;17(2):242-53.

101. Baracat MC, Serafini PC, Simões R dos S, Maciel GA, Soares JM Jr, Baracat EC. Systematic review of cell adhesion molecules and estrogen receptor expression in the endometrium of patients with polycystic ovary syndrome. Int J Gynaecol Obstet 2015;129(1):1-4.

102. Jakubowicz DJ. Seppälä M. Jakubowicz S. RodriguezArmas O. Rivas-Santiago A. Koistinen H. Nestler J. E. Insulin Reduction with Metformin Increases Luteal Phase Serum Glycodelin and Insulin-Like Growth Factor-Binding Protein 1 Concentrations and Enhances Uterine Vascularity and Blood Flow in the Polycystic Ovary Syndrome 1. J Clin Endoc Metabol 2001;86(3): 1126-33.

103. Talbi S. Hamilton AE. Vo KC. Tulac S. Overgaard MT. Dosiou C. Giudice LC. Molecular phenotyping of human endometrium distinguishes menstrual cycle phases and underlying biological processes in normo-ovulatory women. Endocrin 2006;147(3):1097-1121.

104. Zhou J. Dsupin BA. Giudice LC. Bondy CA. Insulin-like growth factor system gene expression in human endometrium during the menstrual cycle. J Clin Endoc Metabol 1994;79(6);1723-34.

105. Giudice LC. Dsupin BA. Irwin JC. 1 Steroid and peptide regulation of insulin-like growth factor-binding proteins secreted by human endometrial stromal cells is dependent on stromal differentiation. J Clin Endoc Metabol 1992; 75(5):1235-41.

106. Lathi RB. Hess AP. Tulac S. Nayak NR. Conti M. Giudice LC. Dose-dependent insulin regulation of insulin-like growth factor binding protein-1 in human endometrial stromal cells is mediated by distinct signaling pathways. J Clin Endoc Metabol 2005;90(3):1599-1606.

107. Schulte MM. Tsai JH. Moley KH. Obesity and PCOS The Effect of Metabolic Derangements on Endometrial Receptivity at the Time of Implantation. Reprod Scien 2015:22(1):6-14

108. Matteo M. Serviddio G. Massenzio F. Scillitani G. Castellana L. Picca G. Liso A. Reduced percentage of natural killer cells associated with impaired cytokine network in the secretory endometrium of infertile women with polycystic ovary syndrome. Fertil Steril 2010;94(6):2222-7.

109. Piltonen TT. Chen JC. Khatun M. Kangasniemi M. Liakka A. Spitzer T. Giudice LC. Endometrial stromal fibroblasts from women with polycystic ovary syndrome have impaired progesterone-mediated decidualization, aberrant cytokine profiles and promote enhanced im- 
mune cell migration in vitro. Hum Reprod 2015;30(5):

110. Huang Y. Zhao Y. Yan L. Chuai YH. Liu LL. Chen Y. Wang AM. Changes in Coagulation and Fibrinolytic Indices in Women with Polycystic Ovarian Syndrome Undergoing Controlled Ovarian Hyperstimulation. Int J Endoc 2014 DOI 10.1155/2014/731498

111. Devroey P. Bourgain C. Macklon NS. Fauser BC. Reproductive biology and IVF: ovarian stimulation and endometrial receptivity. Trend Endoc Metabol 2004; 15(2):84-90.
112. Sharara FI. McClamrock HD. High estradiol levels and high oocyte yield are not detrimental to in vitro fertilization outcome. Fertil Steril 1999; 72(3):401-5.

113. Valbuena D. Martin J. de Pablo JL. Remohí J. Pellicer A. Simón C. Increasing levels of estradiol are deleterious to embryonic implantation because they directly affect the embryo. Fertil Steril 2001;76(5):962-8.

114. Okon MA. Laird SM. Tuckerman EM. Li TC. Serum androgen levels in women who have recurrent miscarriages and their correlation with markers of endometrial function. Fertil Steril 1998;69(4):682-90. 